\title{
Long-Term Outcomes of Single Stage Dorsal Onlay Buccal Mucosa Urethroplasty for Different Anterior Urethral Strictures:A Prospective Study
}

\begin{abstract}
Objective: To report detailed technique and results of one stage dorsal onlay buccal mucosa graft for repair of anterior urethral strictures.

Patients and methods: A total of 45 males with anterior urethral stricture $\geq 2.5 \mathrm{~cm}$ not amenable for excision and primary anastomosis. All patients underwent one-stage dorsal onlay buccal mucosa urethroplasty. Their ages ranged from 14 to 75 years (mean of 35.5 yr). A successful outcome means normal voiding pattern without any post-operative intervention

Results and limitations: The stricture was iatrogenic in 15 (33.3\%) cases, idiopathic in $12(26.7 \%)$, traumatic in $10(22.2 \%)$ cases, and post inflammatory in $8(17.8 \%)$ cases. The mean operative time was 158 minutes. The mean stricture length was $5.6 \mathrm{~cm}$ (range: 2.5 $16 \mathrm{~cm}$ ), while, the mean graft length was $7.2 \mathrm{~cm}$ (range: $4.5-18 \mathrm{~cm}$ ). The mean followup period was 36 months (range 27-48). Overall success rate was $88.9 \%(40 / 45)$. Four patients developed stricture Recurrence. Two of them had annular short bulbar stricture for which direct vision internal urethrotomy was performed and the other two cases needed redo urethroplasty. One patient had dehiscence of glans penis after repair of pan urethral stricture and repair was required. This case and the previous four cases were considered failures $(11.1 \%)$. The most common complication was urinary tract infection (24.4\%). All patients returned to normal diet within 48 hours without oral complications.
\end{abstract}

Conclusion: Meticulous repair of anterior urethral strictures using dorsal onlay bucca graft is technically easy with high success rate. Buccal graft harvesting is simple and easy procedure.

Keywords: Urethral stricture; Buccal mucosa; Urethroplasty
Volume 5 Issue 5 - 2017

\author{
Osama A Shalkamy, Ahmed F Abdelrahim, \\ Shawky A Elmikkawy, Mourad M Mourad, \\ Sayed M Eleweedy \\ Urology Department,Al-Azhar Faculty of Medicine, Egypt
}

Correspondence: Osama A Shalkamy, Urology Department, Al-Azhar Faculty of Medicine, Nasr city- Cairo-Egypt, Tel 1090085651; Email Dr_shalkamy@yahoo.com

Received: May 06, 2017| Published: December 05, 2017
Abbreviations: BMG: Buccal Mucosa Graft; EPA: Excision and Primary Anastomosis; DVIU: Direct Vision Internal Urethrotomy; IPSS: International Prostate Symptom Score; UTI: Urinary Tract Infection; RUG: Retrograde Urethrogram

\section{Introduction}

Surgical treatment of urethral strictures is a continually evolving process, and currently there is renewed controversy over the best means of urethral reconstruction. Buccal mucosa graft (BMG) has gained much interest because of its excellent short and long-term results, low post-operative complication rate, and relative ease of use. ${ }^{1,2}$ A number of series reported success rate of $87-90 \%$ using ventral grafts with very acceptable associated morbidity. ${ }^{3,4}$ Barbagli et al. ${ }^{5}$ introduced the concept of dorsal BMG and some authors hypothesized that this approach might result in superior results compared with ventral grafting ${ }^{6,7}$ In a recent study, Kumar et al. ${ }^{8}$ confirmed the success of dorsal onlay BMG urethroplasty in long segment anterior urethral stricture extending from external meatus to bulbar urethra. Herein we report detailed techniques and results of one stage dorsal onlay buccal mucosa graft for repair of anterior urethral strictures.

\section{Patients and Methods}

This prospective longitudinal study included 45 male patients with a mean age of $35.5+16$ (range 14-75 years) who diagnosed with anterior urethral stricture $\geq 2.5 \mathrm{~cm}$ not amenable for excision and primary anastomosis (EPA). After approval of the study protocol by the local ethics committee, an informed written consent was signed by all patients enrolled in the study. Exclusion criteria included: History of urethral dilatation or internal urethrotomy within 3 months before planned urethroplasty, patients who had an infectious disease affecting the mouth (e.g. Candida or herpes virus), and patients who had previous surgery in the mandibular arch that prevents the mouth from being opened wide. Thirty patients had a previous history of intervention with direct vision internal urethrotomy (DVIU) in 25, urethroplasty in 4, and urethral stent in one patient. Preoperative evaluation included full clinical assessment including International prostate symptom score (IPSS), urine analysis, urine culture, serum creatinine, uroflowmetry, ultrasound estimation of post-void residual urine, retrograde and voiding cystourethrography. Twenty five Patients $(55.6 \%)$ with positive urine culture were given appropriate antibiotics five days before surgery and continued for one week postoperatively.

Patients with negative culture received broad-spectrum antibiotic with induction of anesthesia and continued one week postoperatively. Then all patients received Nitrofurantoin $100 \mathrm{mg}$ per day until removal of the catheter. Mouth washes with povidone-iodine oral solution were started 2 days prior to surgery and continued postoperatively for 3 days. All patients underwent dorsal on lay buccal graft urethroplasty. After General anesthesia with nasal endotracheal intubation preliminary urethroscopy was performed to all patients to confirm the site of stricture and to pass a guide-wire or ureteric catheter through the lumen for easy identification of the lumen later on. 


\section{In bulbar strictures (27 patients)}

After a perineal skin incision, the bulbospongiosus muscle was separated in the midline and, in patients with proximal urethral strictures; the central tendon of the perineum was dissected. The urethra was mobilized, dissected from the corpora cavernosa and was then rotated 180 degrees. The dorsal urethral surface was incised along the midline and the urethral lumen was exposed. Then the stricture was incised along its entire length, and the incision was extended for about $1 \mathrm{~cm}$ both proximally and distally into the normal urethral lumen. Calibration of proximal and distal urethra was performed to ensure patency. The length of the stricture and width of the remaining urethral plate were accurately measured. A buccal mucosal graft was harvested and prepared according to the stricture length and width. The buccal mucosal graft was sutured, splayed, and quilted over the corpora cavernosa using 5-0 polyglactin interrupted stitches. The two apices of the graft were sutured to the proximal and distal apices of the urethrotomy using 4-0 polyglactin interrupted stitches. A Foley 16 French silicon urethral catheter was inserted. The bulbar urethra was returned to its original position over the graft and the edges of the urethra were sutured to the edges of the graft using running 4-0 polyglactin sutures. A rubber drain was then fixed. The bulbospongiosus muscle was re-approximated over the corpus spongiosum. Colles' fascia, the perineal fat and the skin were closed with interrupted absorbable sutures (Figure 1).

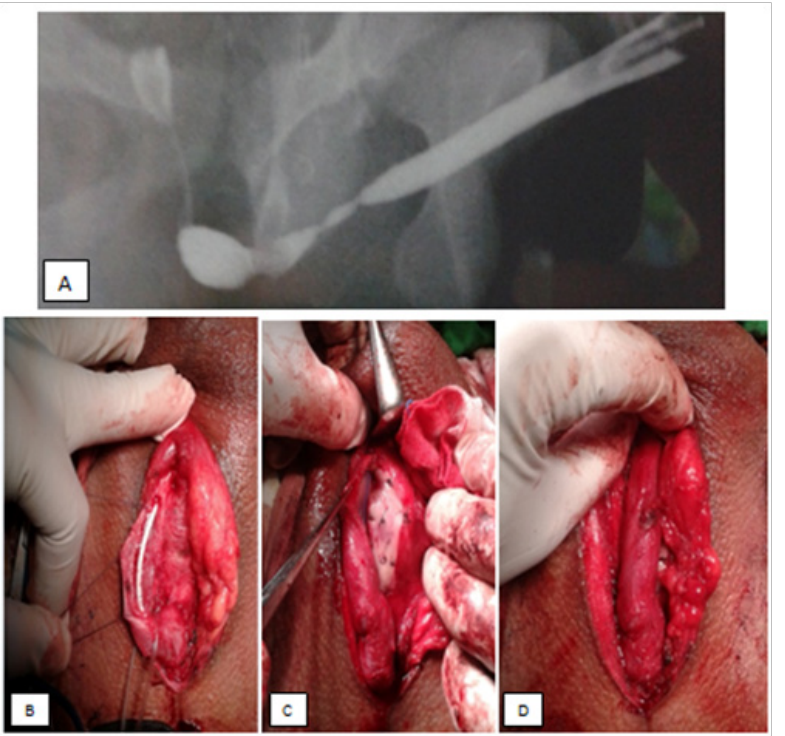

Figure I Buccal mucosal urethroplasty for bulbar stricture, A-RUG. BMobilization, rotation, and dorsal incision of the urethra. C- BMG was sutured, splayed, and quilted over the corpora cavernosa. D- The edges of the urethra were sutured to the edges of the graft.

\section{In bulbopenile and panurethral strictures ( 12 patients)}

Following mobilization of the bulbar urethra, and by invagination of the penis, the penile urethra was dissected. Dorsal urethrotomy followed by grafting was performed as before.

\section{In penile strictures (6 patients)}

After a circumferential incision and degloving of the penis, the urethra was dissected, followed by dorsal urethrotomy and grafting as before. In 2 cases, the penile urethral stricture extended to the external urethral meatus. Meatotomy was done, and then the buccal mucosal graft was sutured, splayed, and quilted over the incised glans.

\section{Buccal mucosal graft harvesting}

In all cases, after preparation of the urethra and recognition of the size of the needed graft, the same team moved to harvest the graft. The donor site was prepared, and then cleaned using a solution containing $10 \%$ povidone-iodine, and the Boyle Davis mouth gag was used to optimize exposure in some patients. Stay suture was applied to the external edge of the graft to keep the oral mucosa stretched. The Stensen's duct was identified and the desired graft size was measured and marked in an ovoid or rectangular shape. Then, $5 \mathrm{ml}$. of $0.5 \%$ adrenaline $(1: 200,000)$ was injected submucosal along the lateral borders of the graft site, to facilitate and enhance hemostasis. The graft was configured according to the desired width and length. The oral mucosal graft was harvested by dissecting the mucosa off the buccinator muscle. The oral mucosal donor site was inspected for bleeding and the defect was closed using a running or interrupted 4-0 polyglactin sutures. When necessary (for longer strictures) an extra graft was taken from the other cheek. The donor site is packed with a piece of gauze which is removed in the ward (Figure 2). The graft was held for defatting over the surgeon's finger, and then the graft was placed in saline with Gentamycin solution and left ready for usage. In all cases, after graft harvesting, the same team rescrubbed and moved to the perineum.

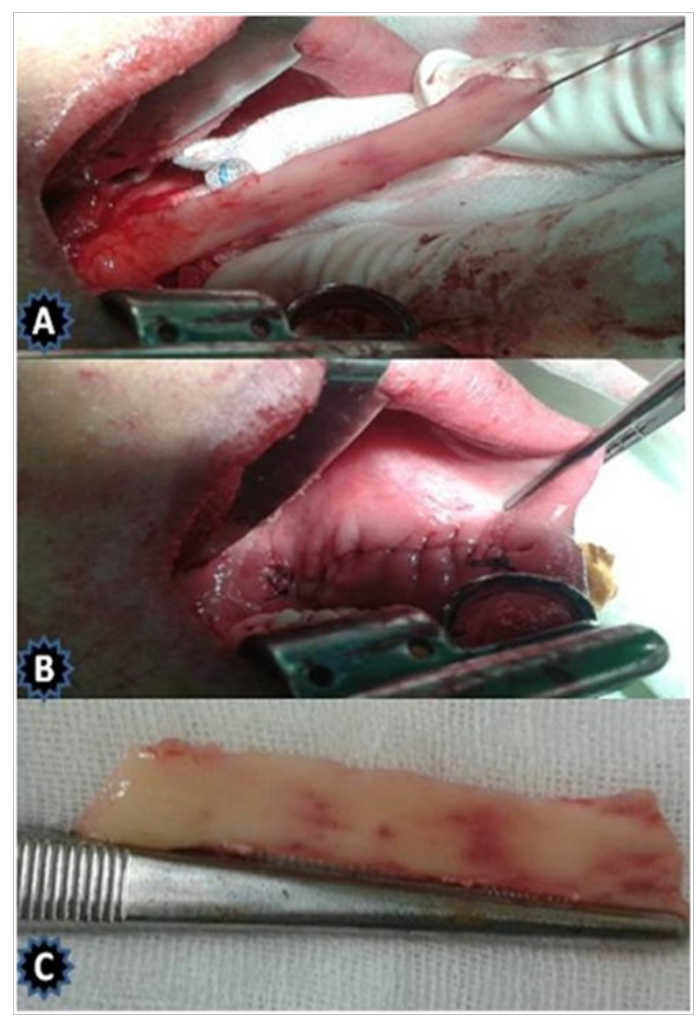

Figure 2 Harvested buccal mucosa.A- BMG harvesting. B- closure of the bed. C-The graft.

Patients were discharged from the hospital 3 days postoperatively. Peri-catheter urethrography was done three weeks post-operatively. Any extravasation dictating extending the period of catheterization for another week. Uroflowmetry and urine culture were done after removal of the catheter and Uroflowmetry was repeated every 4 months in the first year and every 6 month thereafter. When symptoms of decreased force of stream were present and/or the maximum flow rate $(\mathrm{Qmax})$ was $\leq 14 \mathrm{ml} / \mathrm{s}$, retrograde and voiding urethrography 
and urethroscopy were repeated. In all cases retrograde and voiding urethrography was repeated at 12 month. A successful outcome was defined as normal voiding pattern without any intervention postoperatively. Failure was the need for subsequent urethral procedures including dilation.

\section{Results}

Clinical presentation included weak urinary stream in $26(57.8 \%)$ patients, weak urinary stream with recurrent UTI in $8(17.8 \%)$, urine incontinence in $2(4.4 \%)$, and inability to void with suprapubic catheter in $9(20 \%)$. The etiology of urethral stricture was iatrogenic, idiopathic traumatic, and post inflammatory (Table 1). The mean preoperative Qmax in 36 cases was $4.5+2.5 \mathrm{ml} / \mathrm{sec}$ (range: $1.2-7$ ). The mean preoperative IPSS was 25 (range: 16-33). The stricture length ranged from 2.5 to $16 \mathrm{~cm}$ with a mean of $5.6+2.9 \mathrm{~cm}$ (Table 1). The mean preoperative postvoid residual urine in 36 patients was $60.1+73.78 \mathrm{ml}$. Buccal mucosa was harvested from right inner cheek in 42patients (93.3\%) and from both sides in $3(6.7 \%)$. The mean graft length was $7.2+2.9 \mathrm{~cm}$ (range: $4.5-18 \mathrm{~cm}$ ) and the mean graft width was $2+1.6 \mathrm{~cm}$ (range: $1.8-2.5 \mathrm{~cm}$ ). The mean operative time was $158+13$ minutes (range: $140-170$ minutes) with a mean estimated blood loss of $400+82.6 \mathrm{cc}$. All patients returned to normal diet within 48 hours. Four patients developed early oral discomfort which resolved spontaneously within 3 weeks. 37 (82.2\%) patients had no leakage in the peri-catheter urethrogram, and the duration of catheterization was 3 weeks. However, 8 (17.8\%) patients had leakage in the peri-catheter urethrogram, and the catheter duration was extended to 4 weeks.

Table I Characteristics of urethral stricture in 45 patients studied

\begin{tabular}{|l|l|l|}
\hline Characteristics & $\mathbf{N}(\%)$ \\
\hline \multirow{5}{*}{ Etiology } & latrogenic & $15(33.3)$ \\
& Idiopathic & $12(26.7)$ \\
& Trauma & $10(22.2)$ \\
& Post- inflammatory & $8(17.8)$ \\
\hline \multirow{5}{*}{ Site } & Bulbar & $27(60)$ \\
& Bulbopenile & $9(20)$ \\
& Penile & $6(13.3)$ \\
& Pan anterior urethral stricture & $3(6.7)$ \\
\hline \multirow{3}{*}{ Length } & $2.5-5 \mathrm{~cm}$ & $26(57.8)$ \\
& $5-10 \mathrm{~cm}$ & $16(35.5)$ \\
& $>10 \mathrm{~cm}$ & $3(6.7)$ \\
\hline
\end{tabular}

Table 2 Complications of urethroplasty $(n=45)$

\begin{tabular}{|l|l|}
\hline Complications & N (\%) \\
\hline UTI & I I (24.4\%) \\
\hline Wound Infection & $8(17.8)$ \\
\hline Orchitis & $3(6.7)$ \\
\hline Post void dribbling & $2(4.4)$ \\
\hline Weak ejaculation & $2(4.4)$ \\
\hline Temporary fistula & $\mathrm{I}(2.2)$ \\
\hline Total & $27(60)$ \\
\hline
\end{tabular}

The mean follow- up period was 36+5.2 months (range 27-48 months). Postoperative urine culture was positive in $11(24.4 \%)$ patients. The mean postoperative Qmax was $24.6+7.6 \mathrm{ml} / \mathrm{sec}$. The mean postoperative IPSS was 2.58 (range: $0-15$ ). Recurrent stricture was found in four patients. Two of them had short bulbar stricture for which DVIU was performed and the other two cases needed redo buccal graft urethroplasty. One patient had dehiscence of glans penis after repair of pan urethral stricture and he needed repair using skin flap. This case and the previous three cases were considered failures
(11.1\%). So, the overall success rate was $88.9 \%$ (41 patients). The post-operative complications are summarized in Table 2. One patient developed urethrocutaneous fistula at the first radiological followup which closed spontaneously within 3 weeks (in the presence of suprapubic catheter).

\section{Discussion}

The advantages of using oral mucosa in urethroplasty are numerous since it is hairless, easily harvested from one cheek or both cheeks with negligible oral complications, easy to handle in one- or twostage steps, readily available in all patients and resistant to infection. ${ }^{9}$ Histologically, oral mucosa has a thick elastin-rich epithelium, and a thin highly vascular lamina propria that facilitates inosculation and imbibition with minimal inflammatory response and high compatibility with the recipient site. Moreover it is elastic and highly resilient to compression, stretching and shearing forces and is rarely affected by lichen sclerosus disease ${ }^{10}$ Markiewicz and associates, ${ }^{10}$ reviewed the literature from 1966 to 2006 and found that reconstruction for urethral stricture using buccal mucosa was successful in $66.5 \%(418 / 724)$. In 2008, Barbagli and coworkers, ${ }^{11}$ studied retrospectively 375 patients, who underwent one-stage bulbar urethroplasty using either penile skin or oral mucosal grafts and concluded that oral mucosa was superior to skin for one-stage bulbar urethroplasty, with an overall success rate of $82.8 \%$ compared to $59.6 \%$. The overall success rate reported in the literature for one-stage dorsal onlay BMG urethroplasty ranged from $80 \%$ to $100 \%{ }^{7,11}$ The success rate in the present study is $88.9 \%$, which lies in this range.

In our study as well as in others ${ }^{7,12}$ the process of harvesting of BMG was simple, easy, and rapid procedure and did not create a visible scar at donor site. Submucosal infiltration of diluted epinephrine in a concentration of 1 in 200,000 was commonly used to control bleeding and facilitate harvesting the graft. ${ }^{13}$ We used such infiltration in all our cases. There is much debate regarding closure of donor site. Some authors preferred its tight closure after harvesting the graft from single cheek..$^{14,15}$ They found that patients did well with closure of the donor site, but $16 \%$ of their patients had numbness and $32 \%$ had mouth tightness. Others considered tight closure of donor site unnecessary since it may cause perioral numbness and difficulty with mouth opening and suggested that gentle apposition might be helpful to control local bleeding or using fibrin glue or diathermy for hemostasis. ${ }^{16,17}$ In the current study our policy was to close the defect using a running or interrupted 4-0 polyglactin sutures. No patient in our series developed perioral numbness or difficulty with mouth opening.

There is much controversy as to whether to place BMG dorsally or ventrally. Most researchers place it dorsally in penile urethral strictures, and ventrally in the bulbar urethra, or even place it laterally according to the clinical situation. In 1996, Barbagli et al. ${ }^{18}$ and Patterson and Chapple, 2008, ${ }^{12}$ found that dorsal graft placement offered better vascularization with minimal bleeding and reduced risk for graft contraction and urethral diverticula. However, both techniques were reported to have similar results. ${ }^{19}$ In the present study, we placed the BMG dorsally to reduce the complication rate. No urethral diverticulum occurred but fistula occurred in only one patient who was treated conservatively and the fistula closed spontaneously.

The one stage urethroplasty has several advantages: the stricture can be repaired in one setting, no scrotal incision, and direct access to the strictured segment, low restricture rate, and no postoperative chordee formation. However, the single-stage procedure is not 
appropriate for every patient, and improper patient selection can affect the success rates. ${ }^{13}$ We performed all urethroplasties in this series in one stage since it was feasible to do so. In three patients we needed to harvest BMG from both cheek because the strictured segment was long (Pan anterior urethral stricture). In general, complications after buccal urethroplasty were found to be few. ${ }^{12}$ Complications include bleeding, wound infection, facial pain and swelling, injury to Stensen's duct, perioral paresthesia, and restriction in mouth opening in addition to recurrence of the stricture. ${ }^{20}$ In the present study, complications occurred in $60 \%$ of patients $(27 / 45)$. We had no cases of Stensen's duct injury or paresthesia complication in this study. Facial swelling and restrictions in mouth opening were temporary and resolved within 3 weeks.

\section{Conclusion}

Meticulous repair of anterior urethral strictures using dorsal onlay buccal graft is technically easy with high success rate. Buccal graft harvesting is simple and easy procedure.

\section{Acknowledgements}

None.

\section{Conflicts of Interest}

None.

\section{References}

1. El-kasaby AW, Fath-Alla M, Noweir AM, el-Halaby MR, Zakaria W, et al. (1993) The use of buccal mucosa patch graft in the management of anterior urethral strictures. J Urol 149(2): 276-178

2. Andrich DE, Greenwell TJ, Mundy AR (2003) The problems of penile urethroplasty with particular reference to 2 -stage reconstructions J Urol 170(1): 87-89.

3. Kane CJ, Tarman GJ, Summerton DJ, Buchmann CE, Ward JF, et al. (2002) Multi-institutional experience with buccal mucosa onlayurethroplasty for bulbar urethral reconstruction. J Urol 167(3): $1314-1317$

4. Fichtner J, Filipas D, Fisch M, Hohenfellner R, Thüroff JW (2004) Long-term outcome of ventral buccal mucosa onlay graft urethroplasty for urethral stricture repair. Urology 64(4): 648-650.

5. Barbagli G, Palmenteri E, Rizzo M (1998) Dorsal onlay graf urethroplasty using penile skin or buccal mucosa in adult bulbourethral strictures. J Urol 160(4): 1307-1309.

6. Ahmad H, Mahmood A, Niaz WA, Akmal M, Murtaza B, et al. (2011) Bulbar Urethral Stricture repair with Buccal Mucosa Graft Urethroplasty. J Pak Med Assoc 61(5): 440-442.
7. Tavakkoli Tabassi K, Mansourian E, Yarmohamadi A (2011) OneStage Transperineal Repair of Pan-Urethral Stricture With Dorsally Placed Buccal Mucosal Grafts: Results, Complications, and Surgical Technique. Urol J 8(4): 307-312

8. Kumar S, Bansal P, Vijay MK, Dutta A, Tiwari P, et al. (2013) Buccal mucosal graft urethroplasty in long segment anterior urethral stricture is it gold standard? Saudi J Kidney Dis. Transpl 24(1): 115-120.

9. Barbagli G, Vallasciani S, Romano G, , Fabbri F, Guazzoni G, et al (2010) Morbidity of oral mucosa graft harvesting from a single cheek. Eur Urol 58(1): 33-41

10. Markiewicz MR, Lukose MA, Margarone JE, Barbagli G, Miller KS, et al. (2007) The oral mucosa graft: a systematic review. J Urol 178(2): 387-394.

11. Barbagli G, Guazzoni G, Lazzeri M (2008) One-stage bulbar urethroplasty: retrospective analysis of the results in 375 patients. Eur Urol 53(4): 828-833.

12. Patterson JM, Chapple CR (2008) Surgical techniques in substitution urethroplasty using buccal mucosa for the treatment of anterior urethral strictures. Eur Urol 53(6): 1162-1171.

13. Zimmerman WB, Santucci RA (2011) Buccal mucosa urethroplasty for adult urethral strictures. Indian J Urol 27(3): 364-370.

14. Dublin N, Stewart LH (2004) Oral complications after buccal mucosa graft harvest for urethroplasty. BJU Int 94(6): 867-869.

15. Barbagli G, Fabbri F, Romano G, Angelis MD, Lazzeri M (2009) Evaluation of early, late complications and patient satisfaction in 300 patients who underwent oral graft harvesting from a single cheek using a standard technique in a referral center experience. J urol 181(4): 14-18.

16. Wood DN, Allen SE, Andrich DE, Greenwell TJ, Mundy AR (2004) The morbidity of buccal mucosal graft harvest for urethroplasty and the effect of nonclosure of the graft harvest site on postoperative pain. J Urol 172(2): 580-583.

17. Muruganandam K, Dubey D, Gulia AK, Mandhani A, Srivastava A, et al. (2009) Closure versus nonclosure of buccal mucosal graft harvest site: A prospective randomized study on post operative morbidity. Indian J Urol 25(1): 72-75.

18. Barbagli G, Selli C, Tosto A, Palminteri E (1996) Dorsal free graf urethroplasty. J Urol 155(1): 123-126.

19. Barbagli G, Palminteri E, Guazzoni G, Montorsi F, Turini D, et al (2005) Bulbar urethroplasty using buccal mucosa grafts placed on the ventral, dorsal or lateral surface of the urethra: are results affected by the surgical technique? J Urol 174(3): 955-957

20. Bhargava S, Chapple CR (2004) Buccal mucosal urethroplasty: Is it the new gold standard? BJU Int 93(9): 1191-1193. 